which the coherent scattering amplitudes per molecule and the applied corrections due to the impurities are quoted.

The measurements represent an overdetermined system of equations for computing the scattering amplitudes of hydrogen, carbon, and chlorine. The solution leads to the following weighted means for the bound atoms and-in brackets-for the bound nuclei:

$$
\begin{aligned}
& a_{\mathrm{H}}=-3.740 \pm 0.003 \mathrm{fm} \quad(-3.739 \pm 0.003 \mathrm{fm}), \\
& a_{\mathrm{C}}=6.648 \pm 0.003 \mathrm{fm} \quad(6.657 \pm 0.003 \mathrm{fm}), \\
& a_{\mathrm{C} 1}=9.580 \pm 0.002 \mathrm{fm} \quad(9.605 \pm 0.003 \mathrm{fm}) .
\end{aligned}
$$

The values for the nuclei were calculated with a neutron-electron scattering amplitude $a_{n e}=$ $-0.00146 \mathrm{fm} /$ electron (only Foldy interaction).

The new $a_{\mathrm{H}}$ agrees with the old value -3.741 $\pm 0.011 \mathrm{fm}$ tabulated in Ref. 3 , but is much more accurate. The most recent precise experiments led, by means of different methods, to the same results for $a_{C}$ (nucleus), within the errors: 6.650 $\pm 0.009 \mathrm{fm},{ }^{2} 6.660 \pm 0.006 \mathrm{fm},{ }^{8}$ and $6.657 \pm 0.003 \mathrm{fm}$ (present work). This fact is quite encouraging and strengthens the belief in the presented value for $a_{\mathrm{H}}$.

With the new results the following set of parameters for the low-energy $n-p$ system is calculated:

$$
\begin{aligned}
a_{s}=-23.719 \pm 0.013 \mathrm{fm} \text { and } a_{t}= & 5.414 \\
& \pm 0.005 \mathrm{fm} .
\end{aligned}
$$

The change of the old set 1 is mainly due to the new $\sigma_{\mathrm{H}}$ measured in Ref. 2 , where as the result of the present work leads to a further improvement of the accuracy for the low-energy $n-p$ parameters.

${ }^{1} \mathrm{R}$. Wilson, The Nucleon-Nucleon Interaction, Experimental and Phenomenological Aspects (Interscience, New York, 1963).

${ }^{2}$ T. L. Houk, Phys. Rev. C $\underline{3}, 1886$ (1971).

${ }^{3}$ E. Melkonian, Phys. Rev. $\underline{76}, 1744$ (1949).

${ }^{4} \mathrm{~L}$. Koester, unpublished.

${ }^{5}$ In Ref. 2 the scattering amplitude for the atom of $6.623 \mathrm{fm}$ is quoted instead of the amplitude for the nucleus given above.

${ }^{6}$ H. Maier-Leibnitz, Z. Angew. Phys. 14, 738 (1962).

${ }^{7}$ L. Koester, Z. Phys. 198, 187 (1967).

${ }^{8}$ W. Dilg and H. Vonach, Z. Naturforsch. 26a, 442 (1971).

\title{
Superheavy Elements and an Upper Limit to the Electric Field Strength*
}

\author{
Johann Rafelski, Lewis P. Fulcher, $\dagger$ and Walter Greiner \\ Institut für Theoretische Physik der Universität Frankfurt, Frankfurt am Main, Germany
} (Received 9 August 1971)

\begin{abstract}
An upper limit to the electric field strength, such as that of the nonlinear electrodynamics of Born and Infeld, leads to dramatic differences in the energy eigenvalues and wave functions of atomic electrons bound to superheavy nuclei. For example, the $1 s_{1 / 2}$ energy level joins the lower continuum at $Z=215$ instead of $Z=174$, the value obtained when Maxwell's equations are used to determine the electric field.
\end{abstract}

The spectra of atomic electrons offer one means for the identification of superheavy elements. ${ }^{1-3}$ Most of the previous calculations of the atomic structure of these elements have used the relativistic Hartree-Fock-Slater method $^{1}$ and have either used perturbation theory to calculate field corrections ${ }^{2}$ or ignored the question altogether. Accurate calculations of these corrections have not been made, although a method of calculating them, which does not depend upon an expansion in powers of the external field, has been proposed. ${ }^{4}$

Our search for a method of calculating field corrections in superheavy elements has led us to consider the extent to which these corrections are equivalent to an upper limit for the electric field strength. Such an upper limit emerged quite naturally from the considerations of Born and Infeld, who formulated a nonlinear theory of electrodynamics with the express intention of making the self-energy of the electron finite. We show below that the spectra of atomic electrons bound to superheavy elements with nuclear charges $Z \gtrsim 150$ provide a stringent test of the theory of Born and Infeld and, more generally, of similar theories which lead to an upper limit to the electric field strength. Even if superheavy elements cannot be readily produced, enough information could possibly be gathered in the collisions of heavy ions, such as $\mathrm{Pb}$ on $\mathrm{Pb}$ or $\mathrm{Cf}$ on Cf, to decide if this limit exists. In these collisions the adiabatic approximation should have some validity since the velocity of the electrons in the $1 s$ and $2 p$ atomic orbitals is much faster 
than the relative nuclear velocity. Hence, as far as the electrons in the lower atomic orbitals are concerned, ${ }^{5}$ the collisions of $\mathrm{Pb}$ on $\mathrm{Pb}$ and of $\mathrm{Cf}$ on $\mathrm{Cf}$ may simulate superheavy electronic molecules with $Z=164$ and $Z=196$, respectively. Thus a search for the effects of an upper limit to the electric field strength in the elastic scattering of heavy ions would not require a consideration of the complications of nuclear physics. Such experiments may be feasible in one or two years. ${ }^{3}$

The theory of Born and Infeld is based upon the Lagrangian density ${ }^{6}$

$$
\mathscr{L}=\overrightarrow{\mathrm{E}}_{0}^{2}\left[\left(1+\frac{\overrightarrow{\mathrm{B}}^{2}-\overrightarrow{\mathrm{E}}^{2}}{\overrightarrow{\mathrm{E}}_{0}{ }^{2}}-\frac{(\overrightarrow{\mathrm{E}} \cdot \overrightarrow{\mathrm{B}})^{4}}{\overrightarrow{\mathrm{E}}_{0}^{4}}\right)^{1 / 2}-1\right]
$$

where $\vec{B}$ is the magnetic induction, $\vec{E}$ is the electric field, and $\vec{E}_{0}$ is an upper limit to the electric field strength. The quantity $\overrightarrow{\mathrm{E}}_{0}$ is determined by requiring that the mass of the electron is of an entirely electromagnetic origin. Its numerical value is $1.2 \times 10^{18} \mathrm{~V} / \mathrm{cm}$. This Lagrangian density reduces to that underlying Maxwell's equations whenever $\vec{E}$ and $\vec{B}$ are much smaller than $\vec{E}_{0}$. We consider only the case of electrostatics, where the Euler-Lagrange equations reduce to

$$
\nabla \cdot \vec{D}=0 \text {, }
$$

where $\vec{D}=-\partial \mathscr{L} / \partial \vec{E}$ is the electric displacement. Assuming spherical symmetry, the solution of Eq. (2) is $D=e / r^{2}$, where $e$ is the charge of the electron. We have investigated the class of Hamiltonian densities

$$
\mathcal{H}=\frac{\overrightarrow{\mathrm{E}}_{0}^{2}(n)}{8 \pi n}\left[\left(1+\frac{\overrightarrow{\mathrm{D}}^{2}}{\overrightarrow{\mathrm{E}}_{0}^{2}(n)}\right)^{n}-1\right]
$$

which reduces to the theory of Born and Infeld when $n=\frac{1}{2}$. We determine the quantity $\overrightarrow{\mathrm{E}}_{0}(n)$ in Eq. (3) by requiring that the electromagnetic selfenergy is equal to the energy of the rest mass of the electron. The electric displacement satisfies Eq. (2) for every $n$; the relation between $\vec{E}$ and $\vec{D}$ is different in each case, however. The electromagnetic self-energy is finite ${ }^{7}$ if $n \leqslant \frac{3}{4}$. An upper limit to the electric field strength emerges whenever $n \leqslant \frac{1}{2}$. This upper limit is plotted as a function of the parameter $n$ in Fig. 1 . We note that it varies slowly for $-10 \leqslant n \leqslant-1$ and that the value at $n=-10$ is about twice the value at $n=\frac{1}{2}$.

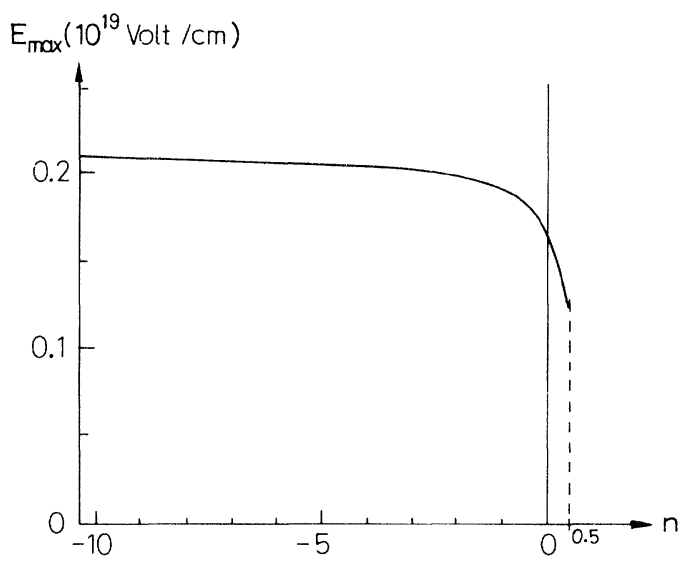

FIG. 1. Maximum value of the electric field as a function of the parameter $n$.

The principle of superposition is no longer valid for the electric field in nonlinear electrodynamics, and thus the treatment of composite systems is more complicated than in the linear case. We consider the electron to move in a spherically symmetric potential due to the nucleus and the other atomic electrons. The calculation of the interaction energy of the electron with the remainder of the atom is based upon the fact that the electric displacement satisfies a linear equation, namely,

$$
\nabla \cdot \overrightarrow{\mathrm{D}}(r)=\rho(\overrightarrow{\mathrm{r}}),
$$

where $\rho$ is the external charge distribution. The nucleus is taken to be a uniformly charged sphere of radius $C=1.2 A^{1 / 3} \mathrm{fm}$, where $A$ is the atomic number. We assume that $A$ is related to the nuclear charge as follows ${ }^{2}$ :

$$
A \cong 0.00733 Z^{2}+1.30 Z+63.6 \text {. }
$$

The electron is taken to be a point charge located a distance $R$ from the center of the nucleus, which we choose as our origin of coordinates. The electric displacement is then the sum of the electron's contribution,

$$
\overrightarrow{\mathrm{D}}_{1}=e(\overrightarrow{\mathrm{r}}-\overrightarrow{\mathrm{R}}) /|\overrightarrow{\mathrm{r}}-\overrightarrow{\mathrm{R}}|^{3},
$$

and the contribution from the nucleus and the remaining electrons,

$$
\overrightarrow{\mathrm{D}}_{2}=\left(\overrightarrow{\mathrm{r}} / r^{3}\right) \int_{0}^{r} \rho^{\prime}\left(r^{\prime}\right) r^{\prime 2} d r^{\prime},
$$

where $\rho^{\prime}\left(r^{\prime}\right)$ includes both the nuclear charge distribution and that of the remaining atomic electrons. The interaction energy for the theory of Born and Infeld is given by

$$
V(\overrightarrow{\mathrm{R}})=\frac{\overrightarrow{\mathrm{E}}_{0}^{2}}{4 \pi} \int d^{3} r\left[\left(1+\frac{\left(\overrightarrow{\mathrm{D}}_{1}+\overrightarrow{\mathrm{D}}_{2}\right)^{2}}{\overrightarrow{\mathrm{E}}_{0}^{2}}\right)^{1 / 2}-\left(1+\frac{\overrightarrow{\mathrm{D}}_{1}^{2}}{\overrightarrow{\mathrm{E}}_{0}^{2}}\right)^{1 / 2}-\left(1+\frac{\overrightarrow{\mathrm{D}}_{2}^{2}}{\overrightarrow{\mathrm{E}}_{0}^{2}}\right)^{1 / 2}+1\right]
$$




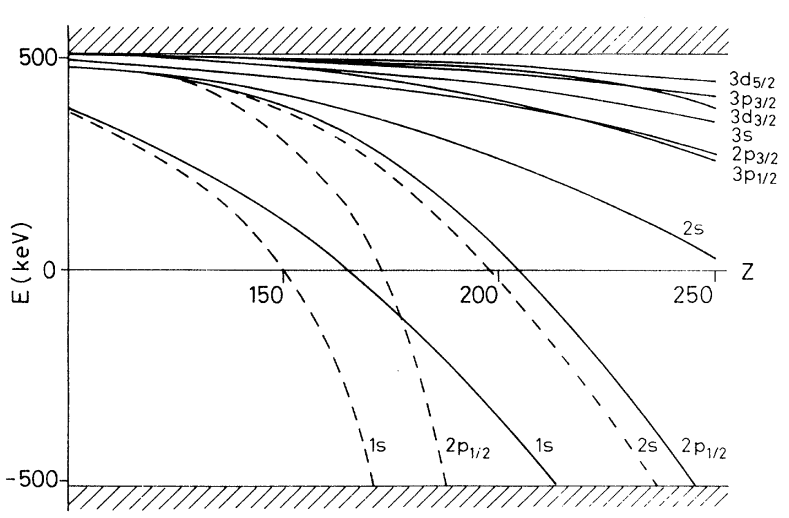

FIG. 2. Energy eigenvalues as a function of nuclear charge. The solid curves have been obtained with the theory of Born and Infeld. The dashed curves have been obtained with Maxwell's equations.

which is readily evaluated numerically. The Thomas-Fermi model of the atom is used to determine the atomic charge distribution.

The energy eigenvalues are determined by solving the Dirac equation numerically for the potential energy of Eq. (8). Several of the lowest eigenvalues are plotted as a function of $Z$ in Fig. 2, where results based on Maxwell's equations are also displayed. The differences are considerable. For example, at $Z=164$ the value obtained for the $1 s$ energy with the theory of Born and Infeld is $270 \mathrm{keV}$ higher than that obtained with Maxwell's equations, and the two values for the $2 p_{1 / 2}$ energy differ by $170 \mathrm{keV}$. Hence the energies of the $2 p_{1 / 2}-1 s$ x rays differ by $100 \mathrm{keV}$ at $Z=164$. The $1 \mathrm{~s}$ level joins the lower continuum at $Z=215$ instead of at $Z=174$. The results shown in Fig. 2 are for a neutral atom. We have done similar calculations for atoms that are completely ionized. The curves for the $1 s$ and $2 p_{1 / 2}$ states are lowered by 5 to $10 \%$ for $150 \leqslant Z \leqslant 200$. Thus the nonlinear effects are considerably larger than the effects due to the other atomic electrons in this region.

The probability densities for an electron in the $1 s$ state are shown in Fig. 3 for $Z=100,114$, and 164. For $Z=164$ the density obtained with Born and Infeld's theory peaks at $110 \mathrm{fm}$ and that obtained with Maxwell's equations peaks at $33 \mathrm{fm}$.

We have estimated the transition probabilities

$$
\begin{aligned}
& W\left(2 p_{1 / 2} \rightarrow 1 s\right) \simeq 9 \times 10^{16} \mathrm{sec}^{-1}, \\
& W\left(2 p_{3 / 2} \rightarrow 1 s\right) \approx 1 \times 10^{17} \mathrm{sec}^{-1}
\end{aligned}
$$

for $Z=1.64$. A calculation of the probability that the $1 s$ state is vacant during a heavy-ion collision is in progress. ${ }^{5}$ If the fission half-lives of superheavy nuclei in the vicinity of $Z=164$ (a possible island of stability ${ }^{8}$ ) are as long as $10^{-16}$ sec, then the $2 p-1 s \times$ rays may be observable in spite of the many $x$ rays which inevitably accompany the fission process.

Lastly, we should like to point out that the measurements of the Lamb shift in light elements are not sufficiently accurate ${ }^{9}$ to detect these nonlinear effects. Also, the change of the $2 p-1 s$ transition energy due to nonlinearities is, e.g., for electronic lead about $0.25 \mathrm{keV}$ and for muonic lead about $30 \mathrm{keV}$. Both effects are thus of the order of magnitude of the vacuum polarization correction and too small to be detected. Such small changes can always be attributed to slight modifications in the nuclear charge distribution. The
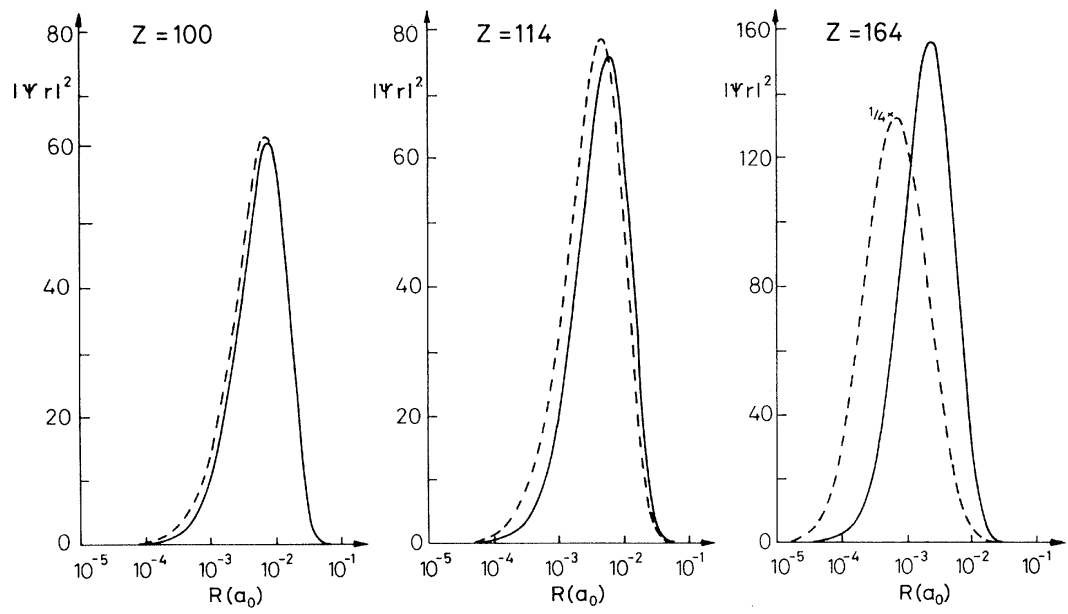

FIG. 3. Probability densities for the $1 s$ electron. The solid curves have been obtained with the theory of Born and Infeld. The dashed curves have been obtained with Maxwell's equations. The unit of the abscissa is the Bohr radius. 
spectra of electrons bound to superheavy nuclei, however, may offer a more stringent test of nonlinear electrodynamics than high-energy electron-electron scattering experiments, ${ }^{9}$ where our inability to quantize nonlinear theories hinders a straightforward comparison of theory and experiment.

We gratefully acknowledge conversations with Professor A. Klein, Professor G. W. Erickson, and Mr. B. Müller. We wish to thank Professor E. Kankeleit for drawing our attention to the elastic scattering of heavy ions below the Coulomb barrier, where nonlinear electrodynamic effects might also be important.

* This work has been supported by the Deutsche Forschungsgemeinschaft and by the Bundesministerium für Wissenschaft und Bildung. $\dagger$ Fellow of the Alexander von Humboldt-Stiftung.

${ }^{1}$ B. Fricke W. Greiner, and J. T. Waber, Theor. Chim. Acta 21, 235 (1971); T. C. Tucker et al., Phys. Rev. 174, 118 (1968); J. B. Mann and W. R. Johnson, Phys. Rev. A $\underline{4}, 41$ (1971).

${ }^{2}$ W. Pieper and W. Greiner, Z. Phys . 218, 327 (1969).

${ }^{3} \mathrm{~F}$. Stephans, private communication.

${ }^{4}$ P. G. Reinhard, W. Greiner, and H. Arenhövel, Nucl Phys. A166, 173 (1971).

${ }^{5}$ Such calculations are presently performed bv Bernd Müller, Institut für Theoretische Physik der Universität Frankfurt.

${ }^{6}$ M. Born and L. Infeld, Proc. Roy. Soc., Ser. A $\underline{144}$, 425 (1934).

${ }^{7}$ J. Rafelski, O. Fulcher, and W. Greiner, to be published.

${ }^{8}$ J. Grumann, U. Mosel, B. Fink, and W. Greiner, Z. Phys. 228, 371 (1969).

${ }^{9}$ S. J. Brodsky and S. D. Drell, Annu. Rev. Nucl. Sci. 20, 147 (1970).

\title{
Charge-Exchange and/or Knockout Spectator Poles in the Reaction $\mathbf{D}\left({ }^{3} \mathrm{He}, t p\right) p$
}

\author{
R. E. Warner* $\dagger$ \\ Physics Department, Oberlin College, Oberlin, Ohio 44074 \\ and
}

G. C. Ball, W. G. Davies, A. J. Ferguson, and J. S. For ster

Chalk River Nuclear Laboratories, Atomic Energy of Canada Limited, Chalk River, Ontario, Canada

(Received 27 August 1971)

\begin{abstract}
Intense spectator-proton peaks were observed in the reaction $\mathrm{D}\left({ }^{3} \mathrm{He}, t p\right) p$. Their shapes were well fitted by plane-wave Born-approximation calculations, assuming either knockout or ${ }^{3} \mathrm{He}-n$ quasielastic scattering accompanied by charge exchange to be the mechanism. Their intensities relative to the quasielastic peaks from $\mathrm{D}\left({ }^{3} \mathrm{He},{ }^{3} \mathrm{He} p\right) n$ were correctly predicted by charge-exchange calculations, but only when an unrealistic mixture of exchange forces was used. All direct knockout calculations gave relative intensities at least ten times too small.
\end{abstract}

This paper reports, for the first time, intense spectator peaks from the reaction $\mathrm{D}\left({ }^{3} \mathrm{He}, t p\right) p$, which may result from quasielastic scattering (QES) accompanied by charge exchange (CE). Quasielastic scattering was first observed by Kuckes, Wilson, and Cooper, ${ }^{1}$ who found that large peaks (called spectator peaks) are observed in the $p-p$ coincidence cross sections from the reaction $\mathrm{D}(p, 2 p) n$ when momentum is transferred only between the two protons, and the neutron (called the spectator particle) remains nearly at rest in the laboratory. QES from the proton in the deuteron has also been studied ${ }^{2-4}$ in the reactions $\mathrm{D}(d, d p) n, D\left({ }^{3} \mathrm{He},{ }^{3} \mathrm{He} p\right) n$, and $\mathrm{D}(\alpha, \alpha p) n$. In CE QES for the reaction $\mathrm{D}\left({ }^{3} \mathrm{He}, t p\right) p$ [see Fig. $1(\mathrm{a})$ ], the ${ }^{3} \mathrm{He}$ and neutron would transfer momentum and exchange charge, emerging as a triton and a proton, and the proton from the deu- teron would remain nearly at rest. Alternatively, a direct knockout (KO) process [see Fig. 1(b)] might also produce spectator peaks.

A CD ${ }_{2}$ target was bombarded with $27-\mathrm{MeV}^{3} \mathrm{He}^{++}$ ions from Chalk River's model MP tandem accelerator. Coincidence events from two $\triangle E-E$ counter telescopes, coplanar with and on opposite sides of the beam, were recorded on magnetic

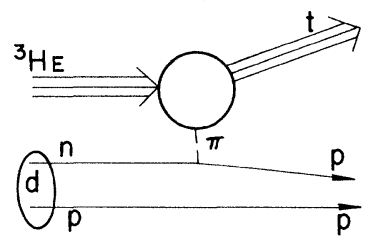

(a)

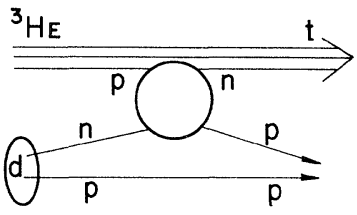

(b)
FIG. 1. Feynman diagrams for (a) charge-exchange quasielastic scattering and (b) knockout. 